\section{Hepatitis C: new clues to better vaccines?}

\author{
Marian E Major
}

$\mathrm{HCV}$ is a major public health problem in the USA and worldwide with the majority of acute infections progressing to chronicity in the absence of therapy. HCV infection is recognised as one of the major causes of liver disease, and is associated with the majority of liver transplantations in most countries. There have been tremendous advances in antiviral drug treatment for HCV over recent years, and current therapies can clear infections in the vast majority of people treated. ${ }^{1}$ However, treatment success can be limited by a range of factors, not least of which include the cost of therapy, which makes access to treatment challenging for many both within the USA and elsewhere. For these reasons, safe, effective and affordable prophylactic vaccines against $\mathrm{HCV}$ remain the best long-term hope for bringing the global epidemic under control. Such vaccines will also help to circumvent issues such as drug resistance, drug-drug interaction in patients with HIV coinfection, susceptibility of treated individuals to reinfection and drug side effects.

There is strong evidence that an effective $\mathrm{HCV}$ vaccine to prevent chronic infection is attainable. A form of protective immunity has been demonstrated in studies of both chimpanzees and humans that have been exposed to the virus after recovering from primary infections. ${ }^{2} 3$ Although HCV RNA can be detected in these secondary infections, the kinetics are different due to rapid control of viral replication, which generally results in lower titres and faster clearance. However, there remain gaps in our knowledge and understanding of the ideal immune response that needs to be induced by a vaccine and the best way to predict the success of a vaccine based on immune markers.

A role for neutralising antibodies in HCV protection should not be excluded. However, many studies have demonstrated the importance of T-cell responses for viral clearance, and as such T-cell-based vaccines have been considered viable candidates for HCV prevention. A number of

Correspondence to Dr Marian E Major, Laboratory of Hepatitis Viruses, Division of Viral Products, Center for Biologics Evaluation and Research, US Food and Drug Administration, Silver Spring, MD 20993, USA; marian. major@fda.hhs.gov chimpanzee vaccine studies have been performed using a variety of vectors to induce T-cell immunity, mainly against the more conserved non-structural proteins. Despite the effective induction of $\mathrm{HCV}$-specific $\mathrm{T}$-cell responses and modification of viral replication kinetics, in many cases, prevention of chronic infection was not achieved even following homologous challenge. ${ }^{4}$ Further advances in understanding T-cell immunity have indicated that the magnitude of a T-cell response is not necessarily a marker of function, and more in-depth analyses such as cytotoxicity, proliferation or polyfunctionality are important to assess quality. ${ }^{5}$ We also need to consider cross-protective responses if an $\mathrm{HCV}$ vaccine is to be functional worldwide. The majority of vaccine candidates have focused on genotype (GT) 1, despite the prevalence of other GTs in the countries that are most in need of a cost-effective form of HCV control.

In this edition of Gut, von Delft et $a l^{6}$ provide a comprehensive and extensive analysis of cross-reactive T-cell responses against GT1 and GT3 antigenic targets. The publication contains a wealth of data on epitopes recognised by both CD4+ and $\mathrm{CD} 8+$ T-cells from patients with HCV GT3 infection and indicates limited cross-reactivity in both chronic and resolved disease. This work provides important information on T-cell specificity for HCV GT3, a highly neglected strain in terms of T-cell analysis despite being the most prevalent in South Asia and the UK. In addition, it prompts new questions regarding the development of broadly protective prophylactic T-cell vaccines against multiple HCV GTs.

The data presented in this analysis of T-cells from patients with HCV infection complement previous data published by the same group analysing T-cell responses induced in human volunteers by vaccination using viral vectors. ${ }^{7}$ Swadling et $a l^{7}$ used chimpanzee adenovirus priming followed by modified vaccinia Ankara (MVA) boosting to induce $\mathrm{HCV}$-specific T-cells against the non-structural proteins of a GT1b strain. They performed detailed analyses of the responses induced by the vaccine, part of which included studies on cross-reactivity. Their analyses showed that the T-cells induced by vaccination were able to target GTs $1 \mathrm{a}, 3 \mathrm{a}$ and $4 \mathrm{a}$ sequences in addition to GT1b. However, although the breadth of the cross-reactive responses was maintained, the magnitude of the response against the other GTs was reduced compared with those against the $1 \mathrm{~b}$-specific peptides by $40 \%, 70 \%$ and $71 \%$ against GT1a, $3 a$ and $4 a$, respectively.

The difference between the findings of these two studies could simply be due to the magnitude of the specific response. Cross-reactivity in the immunised subjects was tested 1 week after the MVA boost, at the peak of the responses. Not surprisingly, Enzyme-Linked ImmunoSpot (ELIspot) responses were lower in patients with GT3 infection (mean spot forming units (SFU) of $\sim 250 /$ million cells against GT3 peptides) ${ }^{6}$ compared with those in immunised subjects (mean SFU of 2360/million cells against GT1b peptides). ${ }^{7}$ Reductions in reactivity of $40 \%-70 \%$ could certainly render some responses undetectable in patients with $\mathrm{HCV}$ infection. An alternative explanation could be that natural infection mainly induces limited T-cell cross-reactivity, whereas a viral vector vaccine can induce qualitatively different T-cells or present a different repertoire of epitopes. Interestingly, although cross-protection from secondary HCV infections has been demonstrated, when persistent infections have occurred, these have often been the result of secondary infections with heterologous viruses. $^{8} 9$ It may certainly be possible that viral vectors induce qualitatively different memory T-cell responses due to different routes of administration and different forms of antigen processing and presentation; whether this can result in better cross-protection remains to be determined.

The studies by von Delft et $a l^{6}$ prompt some interesting questions and serve to demonstrate the complexity of HCV T-cell studies. How should we assess future vaccine candidates for $\mathrm{HCV}$ clinical trials, and which characteristics will be most helpful in predicting vaccine efficacy? Certainly, cross-reactive responses should be considered. These can be inferred from analyses of sequence conservation of dominant epitopes or T-cell targets. However, the recognition of variant epitopes still needs to be confirmed through experimental assays to gain a full understanding of the functional qualities of the cross-reactive T-cells, and the potential these have for long-term memory. Should we move away from whole antigen vaccines and consider targeting specific conserved epitopes such that vaccines contain sequences covering multiple $\mathrm{HCV}$ GTs? This type of design has been shown to be feasible for an HCV global vaccine. ${ }^{10}$ These questions apply equally to antibody 
and T-cell-based vaccines, and to vaccines for a number of other pathogens such as HIV, dengue virus and Ebola virus. It may seem premature to be discussing ways to generate better $\mathrm{HCV}$ vaccines when we still do not fully understand the best approach for a type-specific vaccine. However, if we do not consider these issues now, we cannot plan the studies needed to address these problems and take HCV vaccine development to the next level.

Disclaimer My comments are an informal communication and represent my own best judgement. These comments do not bind or obligate the FDA.

Competing interests None declared.

Provenance and peer review Commissioned; internally peer reviewed.

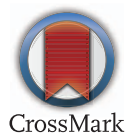

To cite Major ME. Gut 2016;65:4-5.
Received 18 May 2015

Accepted 19 May 2015

Published Online First 19 June 2015

\section{SLinked}

- http://dx.doi.org/10.1136/gutjnl-2014-308724

Gut 2016;65:4-5. doi:10.1136/gutjnl-2015-309829

\section{REFERENCES}

1 Pawlotsky JM, Feld JJ, Zeuzem S, et al. From non-A, non-B hepatitis to hepatitis C virus cure. J Hepatol 2015:62(1S):S87-99.

2 Lanford RE, Guerra B, Chavez D, et al. Cross-genotype immunity to hepatitis $C$ virus. J Virol 2004;78:1575-81.

3 Osburn WO, Fisher BE, Dowd KA, et al. Spontaneous control of primary hepatitis $C$ virus infection and immunity against persistent reinfection. Gastroenterology 2010;138:315-24.

4 Dahari H, Feinstone SM, Major ME. Meta-analysis of hepatitis $C$ virus vaccine efficacy in chimpanzees indicates an importance for structural proteins. Gastroenterology 2010;139:965-74.
5 Seder RA, Darrah PA, Roederer M. T-cell quality in memory and protection: implications for vaccine design. Nat Rev Immunol 2008;8:247-58.

6 von Delft A, Humphreys IS, Brown A, et al. The broad assessment of hepatitis $C$ virus genotype 1 and 3 antigenic targets reveals limited cross-reactivity with implications for vaccine design. Gut 2016;65:112-23.

7 Swadling L, Capone S, Antrobus RD, et al. A human vaccine strategy based on chimpanzee adenoviral and MVA vectors that primes, boosts, and sustains functional HCV-specific T cell memory. Sci Transl Med 2014:6:261ra153.

8 Prince AM, Brotman B, Lee DH, et al. Protection against chronic hepatitis $C$ virus infection after rechallenge with homologous, but not heterologous, genotypes in a chimpanzee model. J Infect Dis 2005;192:1701-9.

9 Schulze Zur Wiesch J, Lauer GM, Timm J, et al. Immunologic evidence for lack of heterologous protection following resolution of HCV in patients with non-genotype 1 infection. Blood 2007;110:1559-69.

10 Yusim K, Fischer W, Yoon H, et al. Genotype 1 and global hepatitis C T-cell vaccines designed to optimize coverage of genetic diversity. J Gen Virol 2010;91(Pt 5):1194-206. 